Braz J Med Biol Res, December 2011, Volume 44(12) 1276-1284

doi: 10.1590/S0100-879X2011007500134

Cardiac autonomic modulation during progressive upper limb exercise by patients with coronary artery disease

H.G. Machado, R.P. Simões, R.G. Mendes, V. Castello, L. Di Thommazo, L.B. Almeida, S.L.B. Lopes, A.M. Catai and A. Borghi-Silva

The Brazilian Journal of Medical and Biological Research is partially financed by

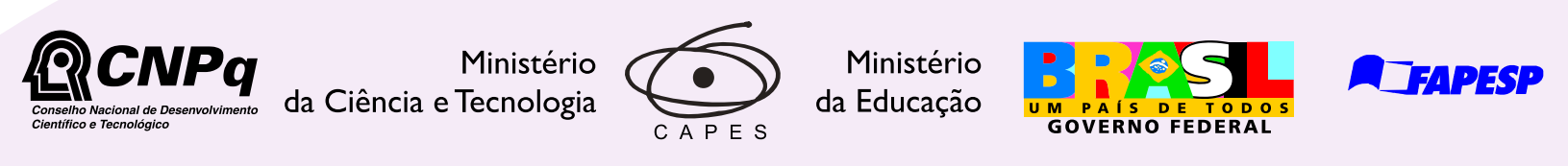

Institutional Sponsors
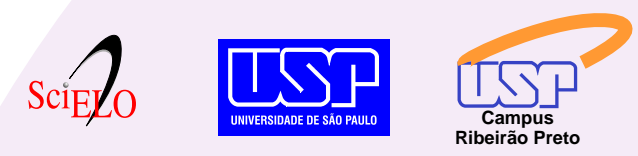

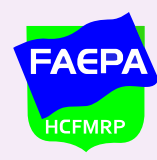

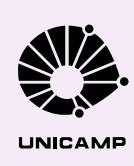

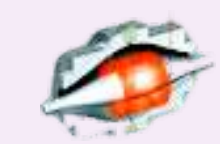

๑ SHIMADZU

Explore High - Performance MS Orbitrap Technology In Proteomics \& Metabolomics

analitica Thermo 


\title{
Cardiac autonomic modulation during progressive upper limb exercise by patients with coronary artery disease
}

\author{
H.G. Machado ${ }^{1}$, R.P. Simões ${ }^{2}$, R.G. Mendes $^{2}$, V. Castello², L. Di Thommazo², \\ L.B. Almeida², S.L.B. Lopes ${ }^{3}$, A.M. Catai ${ }^{2}$ and A. Borghi-Silva1,2 \\ ${ }^{1}$ Programa de Pós-Graduação Interunidades em Bioengenharia, Escola de Engenharia de São Carlos, \\ Universidade de São Paulo, São Carlos, SP, Brasil \\ 2Laboratório de Fisioterapia Cardiopulmonar, Núcleo de Pesquisa em Exercício Físico, \\ Departamento de Fisioterapia, Universidade Federal de São Carlos, São Carlos, SP, Brasil \\ ${ }^{3}$ Departamento de Medicina, Universidade Federal de São Carlos, São Carlos, SP, Brasil
}

\begin{abstract}
The purpose of this study was to investigate the behavior of heart rate (HR) and HR variability (HRV) during different loads of resistance exercise (incline bench press) in patients with coronary artery disease (CAD) and healthy sedentary controls. Ten healthy men ( $65 \pm 1.2$ years, control group, $C G)$ and 10 men with clinically stable CAD (66 \pm 2.4 years, CADG) were recruited. A discontinuous progressive protocol was applied with an initial load of $10 \%$ of the maximum load achieved in the $1 \mathrm{RM}$ (1 repetition maximum) with increase of $10 \%$ until $30 \% 1 \mathrm{RM}$ was reached, which was followed by subsequent increases of $5 \% 1 \mathrm{RM}$ until exhaustion. HRV was analyzed by linear and non-linear methods. There was a significant reduction in rMSSD (CG: $20 \pm 2$ to $11 \pm 3 \mathrm{~ms}$; CADG: $19 \pm 3$ to $9 \pm 1 \mathrm{~ms}$ ) and SD1 indexes (CG: $14 \pm 2$ to $8 \pm 1 \mathrm{~ms}$; CADG: $14 \pm 2$ to $7 \pm 1 \mathrm{~ms}$ ). An increase in HR (CG: $69 \pm 5$ to $90 \pm 5 \mathrm{bpm}$; CADG: $62 \pm 4$ to $75 \pm 4 \mathrm{bpm}$ ) and in systolic blood pressure (CG: $124 \pm 3$ to $138 \pm 3 \mathrm{mmHg}$; CADG: $122 \pm 6$ to $126 \pm 9 \mathrm{bpm}$ ) were observed $(\mathrm{P}<0.05)$ when comparing pre-effort rest and 40\% $1 \mathrm{RM}$ in both groups. Furthermore, an increase in RMSM index was also observed (CG: $28 \pm 3$ to $45 \pm 9$ ms; CADG: $22 \pm 2$ to $79 \pm 33$ ms), with higher values in CADG. We conclude that loads up to 30\% 1RM during incline bench press result in depressed vagal modulation in both groups, although only stable CAD patients presented sympathetic overactivity at 20\% 1RM upper limb exercise.
\end{abstract}

Key words: Dynamic resistance exercise; Coronary artery disease; Heart rate variability; Autonomic nervous system; Upper limb exercise

\section{Introduction}

Resistance exercise (RE) has been considered to be an important component in cardiac rehabilitation programs due to its favorable effects on muscle and cardiac function and quality of life $(1,2)$. The adequate prescription and supervision of RE enhances muscle strength, endurance and functional capacity, and reduces coronary risk factors (1-4). Although RE promotes benefits, it also leads to an overload on the heart and must be carefully prescribed, especially for cardiac patients (5).

Physical exercise has several different characteristics and the type of exercise is identified by taking into account the dominant mechanical and metabolic characteristics. Dynamic-aerobic exercise (endurance) is characterized by limb movement and the predominance of aerobic metabolism. In contrast, dynamic resistance exercise (strength) consists of limb movement in conjunction with load lifting and predominantly anaerobic metabolism (2).

It has been well established that there is a reduction of parasympathetic modulation and an increase of sympathetic modulation at the beginning of dynamic aerobic exercise that results in a heart rate $(\mathrm{HR})$ increase dependent on exercise intensity $(6,7)$. Vagal modulation again becomes predominant during lower intensities of dynamic exercise, with HR and blood pressure gradually stabilizing. However, at higher intensities, sympathetic tonus continues to predominate and results in increased HR and blood pressure until the end

Correspondence: A. Borghi-Silva, Laboratório de Fisioterapia Cardiopulmonar, Núcleo de Pesquisa em Exercício Físico,

Departamento de Fisioterapia, UFSCar, Rod. Washington Luis, km 235, 13565-905 São Carlos, SP, Brasil. Fax: +55-16-3361-2081.

E-mail: audrey@ufscar.br

Received April 12, 2011. Accepted September 27, 2011. Available online October 14, 2011. Published November $28,2011$. 
of the exercise $(6,8)$. A marked increase in blood pressure response has been observed during $\mathrm{RE}$, in contrast to dynamic exercise $(9,10)$. The increase in blood pressure occurs during repetitions, but if the exercise is not sustained, there will be a return to baseline conditions. Moreover, the blood pressure response in resistance exercise depends on the time of muscle contraction and relaxation, since long terms of contraction lead to an increase in peripheral vascular resistance due to mechanical compression of the vessels (11). However, to our knowledge, autonomic nervous control during RE has been little studied.

Specifically, Simões et al. (12) studied the behavior of cardiac autonomic modulation during leg press RE, which is common in fitness centers and rehabilitation programs. They found that systolic blood pressure (SBP), HR and blood lactate were significantly increased, but that $H R$ variability (HRV) indexes such as root mean square of successive differences (rMSSD) and the standard deviation of the instantaneous beat-to-beat R-R interval variability (SD1) (parasympathetic markers) decreased at peak exercise load when compared to resting values. These results support the notion that RE provokes hemodynamic and autonomic modifications that may be due to metabolic alterations in skeletal muscle. However, the autonomic modulation of HR during upper arm RE, which involves less muscle mass, needs to be investigated. In patients with coronary artery disease (CAD), autonomic balance is impaired and HRV is altered due to a decrease in parasympathetic modulation and an increase in sympathetic modulation, which can lead to a high risk of arrhythmias and sudden death (13).

Based on these facts, we hypothesized that the autonomic response of patients with CAD differs from that of healthy individuals during incremental RE due to the autonomic imbalance caused by this disease. Therefore, in order to identify at what percentage of 1 repetition maximum (1RM) cardiovascular response becomes more expressive in upper-limb exercise, the behavior of HRV at increments approaching 1RM was analyzed and evaluated in the present study. An additional purpose was to determine the contribution of increased sympathetic and reduced parasympathetic modulation, as well as that of muscle fatigue, during load increments in RE.

\section{Patients and Methods}

\section{Subjects}

Using the ENE software, version 2.0, the target sample size was calculated to be ten per group considering a $5 \%$ type I error, a 2-sided test, and an $80 \%$ power to detect a significant difference in $\mathrm{HR} \sim 5$ beats, according to a previous study from our laboratory that evaluated healthy and CAD populations under resting condition. Thus, a total of 10 male patients with CAD (CAD group, CADG) and 10 apparently healthy age-matched men, who served as the control group (CG), were recruited for this study after clinical evaluation.
All patients were recruited from the physical therapy clinic of our Institution. The Physiotherapy Laboratory posted an announcement at the university inviting healthy subjects to enroll as control subjects for the study.

The CG was composed of occupationally active subjects who had not participated in a physical training (aerobic or resistance exercise) program prior to the study. For inclusion, the CADG was required to have had an established diagnosis of CAD for at least one year, New York Heart Association (NYHA) classification scores I-II, to have been optimally treated for at least one year according to the American Heart Association/American College of Cardiology recommendations $(1,3)$, to be clinically stable and with controlled blood pressure ( $<130 / 85 \mathrm{mmHg}$ ) (14), considering that 5 CAD patients were taking anti-hypertensive medication. All CAD patients were instructed to maintain the medication prescribed by the cardiologist.

CADG patients had been participating twice a week for at least 12 months in a cardiac rehabilitation program that included only aerobic exercises on a treadmill or cycle. None of the subjects included in the study did regular upper or lower limbs resistance exercises. However, all volunteers (CAD and controls) presented functional capacity between 6 and 8 metabolic equivalents (1) evaluated by clinical exercise testing prior to the proposed protocol. None of the patients were using $\beta$-blockers, verapamil or diltiazen. In the clinical tests, the cardiopulmonary exercise test showed that subjects had a regular aerobic capacity (peak oxygen consumption $\left(\mathrm{VO}_{2}\right)$ between $23-30 \mathrm{~mL} \cdot \mathrm{min}^{-1} \cdot \mathrm{kg}^{-1}$ ) according to the standards of the American Heart Association (15). Moreover, they had no reduced ejection fraction according to two-dimensional echo Doppler cardiography.

Subjects were excluded if they were current smokers or if they presented untreated arterial hypertension or any kind of complex arrhythmia. Further exclusion criteria were the presence of general anemias, metabolic, respiratory or neurological disorders, neuromusculoskeletal disease, or low comprehension capacity. CADG patients were excluded from the study if they had clinical and/or functional evidence of obstructive pulmonary disease (forced expiratory volume in $1 \mathrm{~s}$ and forced vital capacity ratio <0.7), diabetes, anemia, unstable angina or significant cardiac arrhythmias, or a myocardial infarction in the previous 12 months. Healthy sedentary controls were excluded if they were taking any medication. Data regarding subject demographics, anthropometrics and medication are shown in Table 1.

The study was approved by the Ethics Committee on Human Research of the Federal University of São Carlos (\#196/2006). All volunteers were instructed about the experimental procedures and, after agreeing to participate, they signed a written informed consent form.

\section{Clinical examination}

All volunteers underwent clinical evaluation by a cardiologist before the study. This examination consisted of 
anamnesis, 12-lead electrocardiography (ECG), and incremental symptom-limited exercise testing. Blood analyses were performed and hemoglobin, total cholesterol and fractions, triglycerides, fasting glucose, and uric acid were evaluated. One week after clinical evaluation, all subjects underwent cardiopulmonary exercise testing with expired gas analysis (CPX-D, Medical Graphics, USA) in order to evaluate peak oxygen consumption $\left(\mathrm{VO}_{2}\right.$ peak).

\section{Procedures}

The volunteers were physically and anthropometrically evaluated (Welmy scale and stadiometer, Brazil) and body mass index was calculated. The subsequent evaluations (maximum load determination and incremental RE) were performed one week apart.

All experiments were carried out in the morning (to avoid any circadian effects) in a climate-controlled room at $22-24^{\circ} \mathrm{C}$ with $50-60 \%$ relative air humidity. Each volunteer was instructed to wear comfortable clothes, to avoid alcoholic and caffeinated beverages $24 \mathrm{~h}$ prior to the test, to have had a light meal no later than $2 \mathrm{~h}$ before the test and to have performed no exhausting exercise on the day before the test. Immediately prior to data collection, the subjects were interviewed to determine if they had a normal night sleep and their HR and arterial blood pressure were measured to ensure that they were within the normal range $(<130 / 85 \mathrm{mmHg})(14)$. The volunteers were instructed to remain silent during the tests in order to avoid interference with the ECG signal, except to alert the data collector about any kind of symptoms before, during or after the protocol.
Table 1. Demographic and anthropometric data, medications, risk factors, and clinical diagnosis of the control group (CG) and the coronary artery disease group (CADG).

\begin{tabular}{lcc}
\hline & CG $(\mathrm{N}=10)$ & CADG $(\mathrm{N}=10)$ \\
\hline Demographic and anthropometric data & & \\
Age (years) & $65 \pm 1.2$ & $66 \pm 2.4$ \\
Weight $(\mathrm{kg})$ & $70 \pm 2.2$ & $74 \pm 2.5$ \\
Height $(\mathrm{m})$ & $1.66 \pm 0.01$ & $1.67 \pm 0.02$ \\
Body mass index $\left(\mathrm{kg} / \mathrm{m}^{2}\right)$ & $25 \pm 0.8$ & $26 \pm 0.82$ \\
Medications (N) & & \\
Acetylsalicylic acid & - & 9 \\
Anti-arrhythmic & - & 2 \\
ACE inhibitors & - & 5 \\
Calcium-channel blockers & - & 3 \\
Nitrates & - & 1 \\
Antihypercholesterolemics & - & 5 \\
Diuretics & - & 1 \\
Angiotensin II receptor antagonists & - & 1 \\
Risk factors (N) & & 1 \\
Obesity & - & 3 \\
Stress & - & 5 \\
Dyslipidemia & - & 6 \\
Ex-smoker & - & 8 \\
Family history & - & 7 \\
Clinical diagnosis (N) & - & \\
Myocardial infarction & - & \\
Coronary artery bypass grafting & & \\
\hline
\end{tabular}

Data are reported as means \pm SEM. $N=$ number of subjects. ACE $=$ angiotensin-converting enzyme; Ex-smoker = individuals who quit smoking at least one year before the study. No significant difference $(P>0.05)$ between groups (unpaired $t$-test).

\section{RM test}

To determine the protocol loads for incremental RE, the $1 \mathrm{RM}$ test was applied by gradually increasing resistance until the volunteer could successfully complete no more than one repetition on a bench press inclined to $45^{\circ}$ (Vitally Convergent, Brazil). During the test, each volunteer remained seated (with foot support) on the equipment with his trunk inclined to $45^{\circ}$, and was instructed to secure an unattached bar that supported the load. During the movement the elbows and shoulders were flexed and then extended to the initial position. Before the test all volunteers were instructed to avoid the isometric component and the Valsalva maneuver by inhaling during elbow flexion (16). Before the test, a 1-min warm-up was performed with the involved musculature to facilitate motor learning of the exercise technique. The resistance load of $1 R M$ was estimated (1RM-E) before the test by multiplying the body weight of the volunteer by 0.6 , which was based on a previous pilot test. After estimating maximum load, a load of $80 \% 1 \mathrm{RM}-\mathrm{E}$ was applied to start the test. If this was found to be submaximal, $10 \%$ of $1 \mathrm{RM}-\mathrm{E}$ was added after a minimum of 5 min of rest, which allowed $\mathrm{HR}$ and blood pressure to return to base values. If the first attempt was unsuccessful because of excess load, the last $10 \%$ 1RM-E load was removed. Based on the estimated loads of 1RM, it was expected that 1RM could be determined within a maximum of 6 attempts (17). Blood pressure was measured at the end of each attempt and after 5 min of rest before another attempt.

\section{Incremental RE protocol}

The incremental RE protocol was a progressive discontinuous test. The volunteer remained at rest on the equipment for $10 \mathrm{~min}$. Then, the correct technique for executing the proposed exercise was taught. The incremental RE protocol was initiated at a load of $10 \% 1 \mathrm{RM}$, with subsequent increases of $10 \%$ until reaching a load of $30 \% 1 \mathrm{RM}$. Subsequently, the incremental adjustments were continued at 5\% 1RM until exhaustion, with the objective of increasing the number of sets and allowing better visualization of the 
physiological adjustments with increasing load. In each set of exercise, the volunteer performed 36 repetitions at a pace associated with respiratory rhythm: $2 \mathrm{~s}$ of flexion (inspiration) and three of elbow extension (expiration). Respiratory rhythm was maintained as instructed during all loads, as also was correct movement biomechanics. At the end of each set, a recovery period of $15 \mathrm{~min}$ was established so that HR and blood pressure could return to baseline. Loads were increased incrementally until the subject was unable to perform the required number of repetitions within a certain amount of time or until signs or symptoms of intolerance or exhaustion appeared.

During all loads applied in the incremental test, HR was recorded beat-to-beat and the ECG signal was monitored throughout the test. Blood pressure was recorded both at rest and $20 \mathrm{~s}$ after the interruption of effort. Muscle fatigue in the upper limbs was evaluated by a visual Borg scale at the end of each set, with 0 representing no symptoms and 10 representing maximal effort (18).

The criteria for interrupting the load increment protocol were SBP $>200 \mathrm{mmHg}, \mathrm{HR} \geq 85 \%$ of maximum [HR (220 - age $x$ 0.85)] and the development of any potential arrhythmias. If none of the above changes occurred, the subject continued the test until achieving maximum voluntary exhaustion.

\section{Safety and ECG monitoring during the protocols}

During the entire test period, HR was recorded with a HR monitor (Polar Vantage, Finland) linked to an interface (Polar Advantage), which transmitted the data in real time to a microcomputer (Soyo PW 9800 Laptop, USA). Furthermore, the ECG was constantly monitored with a heart monitor (Ecafix TC 500, Brazil) to detect any potential arrhythmias or signs of ischemia that would indicate that the protocol should be interrupted. The appearance of acute electrocardiographic alterations such as atrioventricular block, ST segment depression, or acute arrhythmias (atrial and ventricular ectopy) was monitored. Blood pressure was measured by auscultation (BD sphygmomanometer, Brazil) before and after each maximum load test. The perceived exertion level was obtained with the Borg scale (0-10).

\section{Data analysis}

The data captured by the HR monitor were collected at a sampling frequency of $500 \mathrm{~Hz}$, stored in the computer and visually inspected using the Polar Precision Performance software (version 4.01.029, Finland). The data were then filtered by the same software to avoid contamination of the analysis. The moderate power filter included a protection zone of a minimum of $6 \mathrm{bpm}$. All recorded data presented less than $1.3 \%$ of random error.

The sections selected for HR analysis were collected during the initial rest period with the volunteer positioned on the equipment and during the application of percent $1 \mathrm{RM}$ increments. The most stable section containing at least 2 min of data collected during the 3 min of exercise in each resistance load effort was selected; the initial $40 \mathrm{~s}$ of exercise were excluded because this is the period during which a rapid withdrawal of vagal activity occurs.

The HRV was evaluated with the rMSSD, the root mean square of the differences from the mean interval (RMSM) indexes and the SD1 using Poincaré plotting. The rMSSD and RMSM indexes of the R-Ri were analyzed with a specific routine developed in the Matlab 6.5 software. The rMSSD corresponded to the square root of the sum of the square of the differences between the R-Ri rerecorded, divided by the number of R-Ri within a specified time minus one, which provided information on parasympathetic heart modulation $(19,20)$. The RMSM index corresponded to the square root of the sum of the square of the differences of the individual values in relation to the mean value divided by the number of R-Ri within a specified time and was characterized as a marker of total HRV (7).

Quantitative analysis by Poincaré plotting consisted of plotting each R-Ri as a function of the preceding interval. Through this analysis it was possible to obtain the SD1 index in milliseconds ( $\mathrm{ms}$ ), which provided information on the standard deviation of instantaneous beat-to-beat variability and was characterized as a parasympathetic heart modulation marker $(21,22)$.

\section{Statistical analysis}

Data distribution was determined by the Shapiro-Wilk test and when normality was confirmed the data were reported as means \pm SEM. Two-way ANOVA for repeated measurements was used to compare the variables at different percentages of $1 \mathrm{RM}$, the differences between groups and the values obtained during the rest and the peak conditions. The Tukey post hoc test was used to identify significant differences. The unpaired Student $t$-test was used to compare Borg scale, 1RM load and maximum load achieved values (Table 2).

The probability of a type 1 error was established at $5 \%$ for all tests $(\alpha=0.05)$. Data were analyzed with the Sigma Plot 11.0 software (Systat Software Inc., 2008).

\section{Results}

During the protocol, no signs of complex arrhythmia or ischemia were recorded in either group. Moreover, no patient medications were changed during the study. In the CG, only 2 volunteers presented isolated extra-systoles $(<2 / \mathrm{min})$. In the CADG, 4 subjects presented isolated extrasystoles (<4/min). No symptoms of angina pectoris were reported by the patients.

All 20 volunteers successfully completed the protocol with no abnormalities that would contraindicate enrollment in the present study. As summarized in Table 1, there were no statistically significant anthropometric differences between the two groups of volunteers. 
Table 2. Cardiovascular variables and load obtained during one repetition maximum (1RM) test and heart rate variability indexes obtained at the peak and during the rest of resistance exercise (RE).

\begin{tabular}{|c|c|c|c|c|c|c|c|}
\hline \multirow[t]{2}{*}{ Variables } & \multicolumn{2}{|c|}{ CG $(N=10)$} & \multicolumn{2}{|c|}{ CADG $(N=10)$} & \multicolumn{3}{|c|}{$\mathrm{P}$} \\
\hline & Rest & Peak & Rest & Peak & G & $\mathrm{C}$ & I \\
\hline \multicolumn{8}{|l|}{ 1RM test } \\
\hline $\mathrm{SBP}(\mathrm{mmHg})$ & $124 \pm 3$ & $138 \pm 3$ & $122 \pm 6$ & $126 \pm 9$ & 0.34 & 0.15 & 0.20 \\
\hline $\mathrm{DBP}(\mathrm{mmHg})$ & $76 \pm 1$ & $77 \pm 2$ & $70 \pm 3$ & $72 \pm 3$ & 0.02 & 0.53 & 0.83 \\
\hline $\mathrm{HR}(\mathrm{bpm})$ & $69 \pm 5$ & $90 \pm 5$ & $62 \pm 4$ & $75 \pm 4$ & 0.01 & $<0.001$ & 0.31 \\
\hline Borg - effort (0-10) & 0 & $7 \pm 1$ & 0 & $9 \pm 1$ & 0.01 & - & - \\
\hline Load 1RM (kg) & - & $43 \pm 2$ & - & $38 \pm 3$ & 0.13 & - & - \\
\hline \multicolumn{8}{|l|}{$\mathrm{RE}$} \\
\hline \multicolumn{8}{|l|}{ Cardiovascular variables } \\
\hline $\mathrm{SBP}(\mathrm{mmHg})$ & $121 \pm 4$ & $142 \pm 4$ & $118 \pm 8$ & $158 \pm 10$ & 0.17 & $<0.001$ & 0.04 \\
\hline $\mathrm{DBP}(\mathrm{mmHg})$ & $75 \pm 2$ & $79 \pm 2$ & $69 \pm 3$ & $75 \pm 2$ & 0.02 & 0.04 & 0.62 \\
\hline $\mathrm{HR}(\mathrm{bpm})$ & $66 \pm 3$ & $89 \pm 6$ & $61 \pm 3$ & $86 \pm 4$ & 0.32 & $<0.001$ & 0.78 \\
\hline Borg - effort (0-10) & 0 & $7 \pm 1$ & 0 & $8 \pm 1$ & 0.01 & - & - \\
\hline Maximum load achieved & - & $18 \pm 1$ & - & $15 \pm 2$ & 0.003 & - & - \\
\hline \multicolumn{8}{|l|}{ HRV indexes } \\
\hline rMSSD (ms) & $20 \pm 2$ & $11 \pm 3$ & $19 \pm 3$ & $9 \pm 1$ & 0.50 & $<0.001$ & 0.82 \\
\hline RMSM (ms) & $28 \pm 3$ & $45 \pm 9$ & $22 \pm 2$ & $79 \pm 33$ & 0.08 & $<0.001$ & 0.01 \\
\hline SD1 (ms) & $14 \pm 2$ & $8 \pm 1$ & $14 \pm 2$ & $7 \pm 1$ & 0.58 & $<0.001$ & 0.92 \\
\hline
\end{tabular}

Data are reported as means \pm SEM. $C G=$ control group; $C A D G=$ coronary heart disease group; $S B P=$ systolic blood pressure; $\mathrm{DBP}=$ diastolic blood pressure; $\mathrm{HR}=$ heart rate; $\mathrm{HRV}=$ heart rate variability; $\mathrm{rMSSD}=$ root mean square of successive differences; RMSM = root mean square of the differences from the mean interval; SD1 = the standard deviation (SD) of the instantaneous beat-to-beat R-R interval variability; $G$ = group effect (CG vs CADG); $\mathrm{C}$ = condition effect (rest vs peak); I = interaction between group and condition effects. Statistical analysis was carried out with two-way ANOVA for repeated measurements.

As expected, HR also increased at peak load in both groups when compared to the resting values. However, significantly higher values were detected in the CG (Table 2). Furthermore, the $1 \mathrm{RM}$ test induced a similar significant increase in perceived exertion (Borg scale 0 to 10) in both groups; the resistance load achieved during 1RM was also similar.

Regarding the response to the RE protocol, the criterion for interrupting the protocol for all volunteers was muscle fatigue (Table 2); no test was interrupted due to ECG alterations or excessive SBP or HR. SBP as well as HR increased significantly $(P<0.05)$ in both groups, with no differences between groups.

Both rMSSD and SD1 indexes, which are representative of parasympathetic modulation, presented significant decreases in both groups at peak load compared to resting values. However, regarding the RMSM index, which is representative of total HRV, a significantly higher value was observed in the CADG when comparing at-rest and peak load conditions; a significant intergroup difference was observed at peak load.

All volunteers achieved at least 35\% $1 \mathrm{RM}$ during the RE protocol, which was the maximum load for 1 volunteer in the CADG; 11 volunteers achieved $40 \% 1 \mathrm{RM}$ (5 of the CG and 6 of the CADG), 7 volunteers achieved $45 \% 1 \mathrm{RM}$ (4 of the $\mathrm{CG}$ and 3 of the CADG) and 1 CG volunteer achieved $50 \%$ 1RM. The absolute maximum workload did not differ significantly between groups (Table 2).

Figure 1 shows the behavior of rMSSD, SD1, RMSM, and $H R$ during increased RE loads, starting at resting values and continuing until the common maximum load achieved by most volunteers ( $40 \%$ of $1 \mathrm{RM}$ resistance load). As shown in Figure 1, there was a significant reduction in $\mathrm{rMSSD}$ and SD1 between rest and 30, 35, and 40\% 1RM in both groups (Figure 1). The RMSM index was significantly increased between rest and 20\% 1RM in the CADG, while no significant increase was observed in the CG. At 30, 35, and 40\% 1RM, the rMSSD values were significantly higher in the CADG than in the CG (Figure 1). HR increased significantly between rest and 30\% 1RM in both groups ( $P<0.05)$.

SBP increased significantly between rest and 30\% 1RM in both groups. At $40 \% 1 \mathrm{RM}$ the SBP value at the end of exercise was higher than at rest in both groups, but was significantly higher in the CADG than in the CG, as shown in Figure 2. The diastolic blood pressure values were stable throughout the protocol in both groups (Figure 2). Regarding 

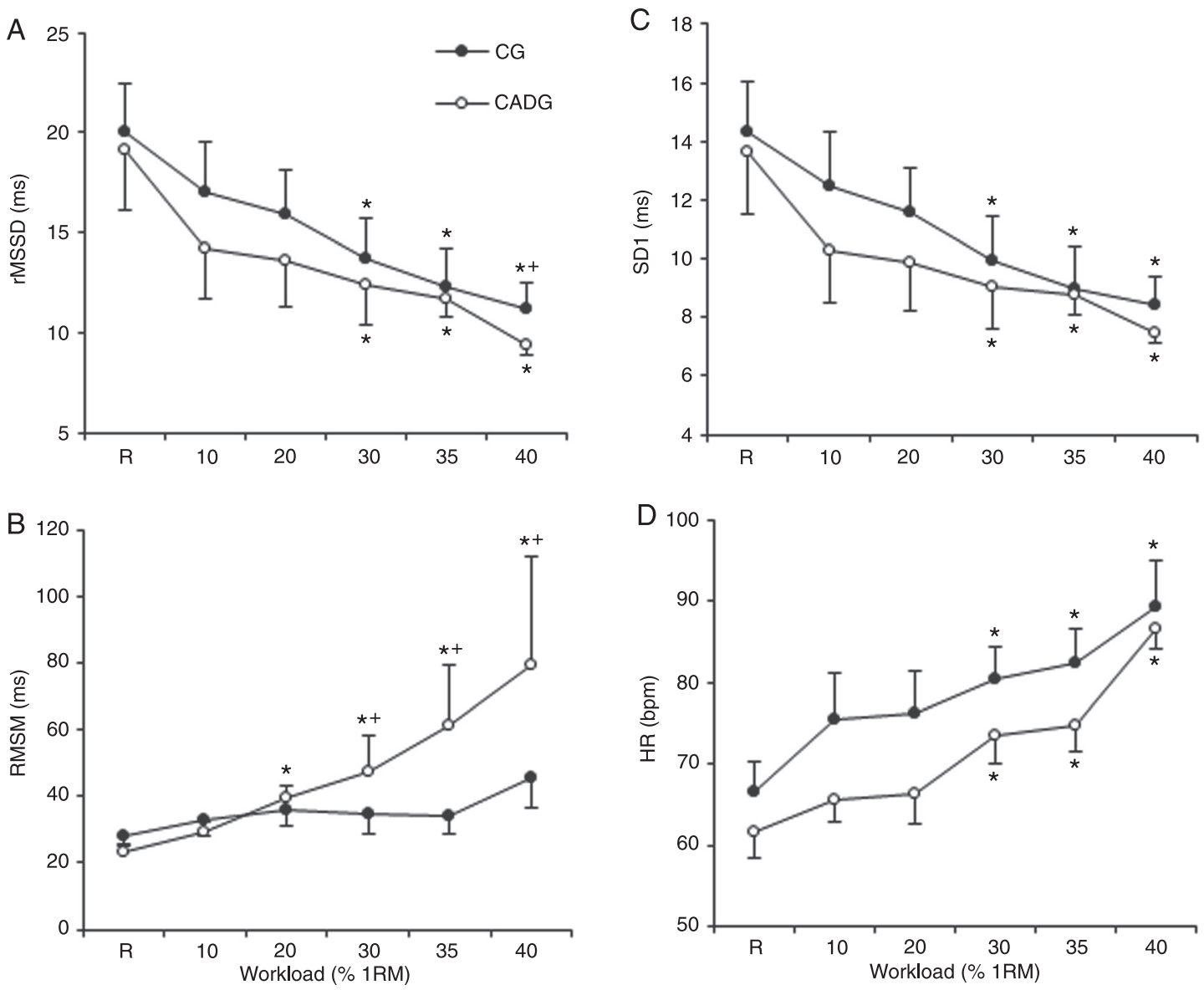

Figure 1. Behavior of variables at resistance increments in percentages of one repetition maximum (1RM). Heart rate variability indexes $(A$, root mean square of successive differences [rMSSD]; $B$, root mean square of the differences from the mean interval [RMSM]; $C$, the standard deviation of the instantaneous beat-to-beat $R-R$ interval variability [SD1]); $D$, heart rate of the control group (CG) and the coronary artery disease group (CADG) during the incremental protocol. Data are reported as means $\pm \mathrm{SEM}$. $\mathrm{R}=$ resting on equipment; HR = heart rate; ${ }^{\mathrm{P}}<0.05$ in relation to the rest condition; ${ }^{+} \mathrm{P}<0.05$ comparing the two groups (two-way ANOVA with repeated measures).

the Borg scale, fatigue symptoms significantly increased beginning at $20 \% 1 \mathrm{RM}$ in both groups. Moreover, the Borg scale results at $40 \% 1 \mathrm{RM}$ were significantly higher in the CADG than in the CG (Figure 2).

\section{Discussion}

The present study investigated the behavior of cardiac autonomic modulation at different percentages of $1 \mathrm{RM}$ in incline bench press RE in individuals with CAD and healthy sedentary age-matched controls. The main findings of this study were: 1) a significant reduction of rMSSD and SD1 indexes, representative of parasympathetic modulation, beginning at 30\% 1RM in both groups; 2) a significant increase in RMSM index, representative of total HRV, beginning at $20 \%$ 1RM in CADG; 3) a significant increase of HR and SBP beginning at $30 \% 1 \mathrm{RM}$ in both groups.
Regarding the safety of the tests, we observed that neither test (1RM and RE) led to inappropriate signs or symptoms or ECG alterations. Specifically regarding the $1 \mathrm{RM}$ test, studies have reported that this type of test is safe and effective for evaluating maximal force in older healthy adults and individuals with CAD $(1,3,23-25)$. Similarly, we observed no ischemic ECG alterations or complex arrhythmic induction during the upper limb exercise. Moreover, the volunteers reported no symptoms (such as angina) and showed no other manifestations of intolerance.

Regarding the cardiovascular response during the dynamic RE protocol, SBP and HR increased significantly in both groups when comparing the rest period to the peak exercise values. Additionally, rMSSD and SD1 indexes decreased in both groups at peak load. The behavior of cardiovascular variables demonstrated that hemodynamic modifications during dynamic exercise might be dependent 

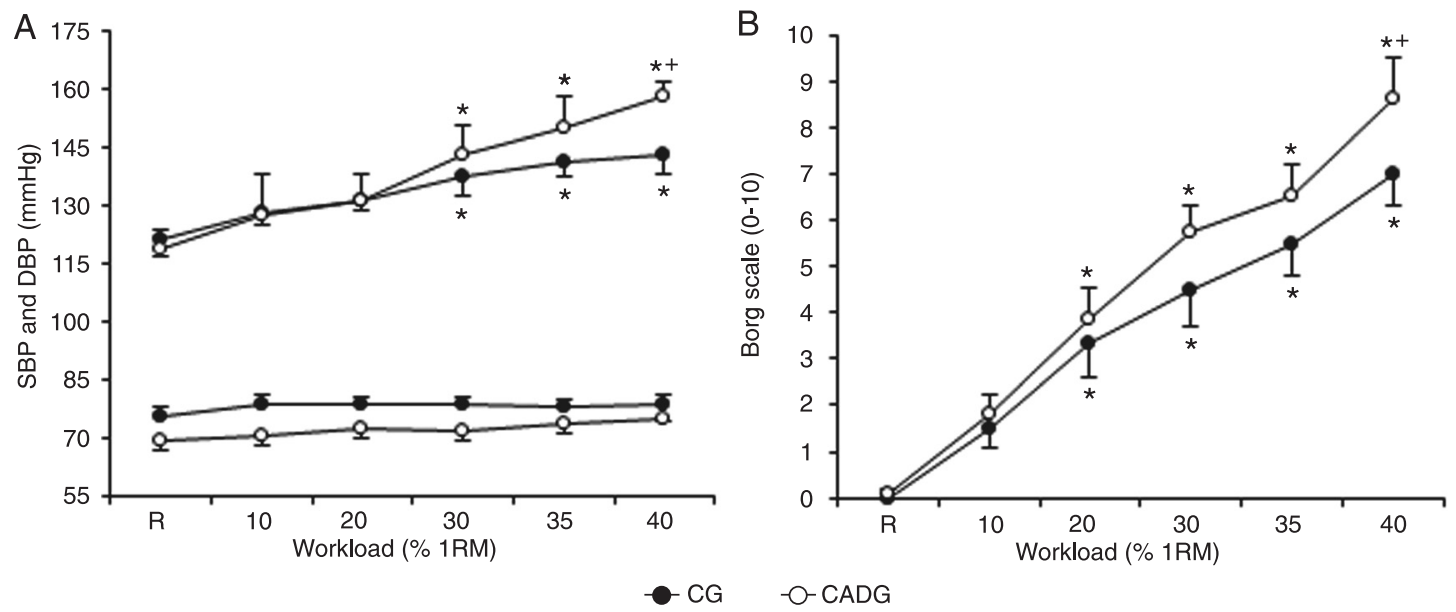

Figure 2. Behavior of $(A)$ systolic blood pressure (SBP) and diastolic blood pressure (DBP), and (B) Borg scale during resistance increments in percentages of 1 repetition maximum (1RM). Data are reported as means \pm SEM. CG $=$ control group; $C A D G=$ coronary artery disease group; $R=$ resting on equipment. ${ }^{*} P<0.05$ in relation to the resting condition; ${ }^{+} \mathrm{P}<0.05$ comparing the two groups (two-way ANOVA with repeated measures).

on both arterial flow to the musculature involved in the effort and the magnitude of resistance (1RM percentage) $(26,27)$.

The rMSSD and SD1 indexes reflect the vagal modulation of $\operatorname{HR}(9,22,28)$. The progressive decrease in this index beginning at $30 \% 1 \mathrm{RM}$ in both groups can be explained by a decrease in parasympathetic modulation and, consequently, a predominance of sympathetic modulation at higher intensities (6). Similarly, Simões et al. (12) observed a significant decrease in SD1 and rMSSD indexes beginning at 30\% $1 \mathrm{RM}$ in leg resistance exercise that was related to lactate accumulation. They concluded that this intensity $(30 \%$ of $1 \mathrm{RM}$ ) was associated with the point at which aerobic metabolism becomes supplemented by anaerobic metabolism. Other investigators have observed that a prevalence of sympathetic modulation of the heart occurs after the anaerobic threshold point has been reached (29). On this basis, our results suggest that parasympathetic modulation tended to be reduced with increasing loads and became more expressive from $30 \% 1 \mathrm{RM}$.

The RMSM index reflects both vagal and sympathetic modulation $(7,20)$ and some investigators consider this index to represent the sympathetic limb of the autonomic nervous system (30). In the present study, the RMSM index increased after 20\% 1RM in the CADG (Figure 1), a fact that may suggest sympathetic overmodulation. It is well accepted that autonomic modulation of HR during exercise is mediated by a central command, exercise pressor reflex and arterial baroreflex (31-33). However, the findings of Koba et al. (34) suggest that an intensive activation of the central command occurs in myocardial infarction (MI) and leads to an excessive sympathetic outflow. In this context, after acute $\mathrm{Ml}$ there is a prevalence of sympathetic tonus, which is similar to our finding that suggested overactivity of sympathetic modulation in individuals who suffered MI (CADG). In the CG, the RMSM index tended to increase, a fact that may suggest an increase in sympathetic modulation at a lesser intensity than that observed in the CADG.

Sympathetic predominance is conspicuous during elevated loads and this may explain the increase in SBP and $\mathrm{HR}$ beginning at 30\% 1RM. This mechanism depends on muscle tension, especially the concentric phase of contraction, which mechanically compresses the peripheral arterial system and causes a decrease in blood flow. The mechanical and metabolic changes can stimulate the afferent fibers of type III and IV musculatures (6). The metabolic changes combined with reduced blood flow can stimulate type IV fibers, which send information to the ventrolateral medullar region, triggering an increase in sympathetic discharge to the cardiovascular system (35). Thus, an increase in HR and blood pressure occurs to restore muscle blood flow, which depends on the intensity and the size of the muscle mass involved in the effort (36).

Upper limb RE led to an increase in perceived exertion that may be explained by the longer duration of each load and the concomitant metabolic changes (37-39), which should be investigated in future studies. Throughout the study protocol, the Borg scale was applied to determine perceived exertion and muscle fatigue. Muscle fatigue was more prominent beginning at 20\% 1RM in both groups and caused the volunteers to interrupt the exercise.

However, due to the methodological considerations and the nature of the protocol, we considered it important to test whether fatigue could interfere in HRV and symptoms. Our results show that even in long protocols, low-to-moderate intensities of upper limb exercise were feasible and did not 
induce undesirable signs or symptoms, even in patients with clinically stable CAD.

Some limitations of this study should be mentioned. First, the proposed protocol had a relatively long duration (36 repetitions), which may have induced the volunteers to reach peak effort at relatively low loads. However, a substantial period of data collection was necessary to capture the HR signal and a greater number of points for the application of methods for HRV analysis, in accordance with Task Force standards (20). Thus, the protocol used in this study was not based on the recommended protocols for individuals with CAD, but was designed as a first attempt to analyze the responses of physiological and autonomic control of HR during upper limb RE in this population. Also, the number of repetitions was high and might have caused a cumulative effect during the loads, but the interval between sets was sufficiently long for a return to baseline levels (40). Further studies are needed to contrast short and long durations of this type of RE on HRV.

Second, we studied just one type of RE that involves only the upper limb muscle groups. However, for a better understanding of physiological responses during RE, studies comparing HRV in upper and lower limbs will be necessary. Third, as blood pressure was measured by the auscultation method after the effort, values may have been underestimated (6). However, as the method of measurement and the time delay after exercise were constant in all experimental conditions, this aspect should not have affected the study comparisons. Furthermore, although invasive methods for measuring blood pressure are reliable, they are expensive, painful and rarely used in conjunction with RE sessions. Finally, we were concerned about the impact that some medications might have on HRV during

\section{References}

1. Pollock ML, Franklin BA, Balady GJ, Chaitman BL, Fleg JL, Fletcher B, et al. AHA Science Advisory. Resistance exercise in individuals with and without cardiovascular disease: benefits, rationale, safety, and prescription: An advisory from the Committee on Exercise, Rehabilitation, and Prevention, Council on Clinical Cardiology, American Heart Association; Position paper endorsed by the American College of Sports Medicine. Circulation 2000; 101: 828-833.

2. Hunter GR, McCarthy JP, Bamman MM. Effects of resistance training on older adults. Sports Med 2004; 34: 329-348.

3. Williams MA, Haskell WL, Ades PA, Amsterdam EA, Bittner $\mathrm{V}$, Franklin BA, et al. Resistance exercise in individuals with and without cardiovascular disease: 2007 update: a scientific statement from the American Heart Association Council on Clinical Cardiology and Council on Nutrition, Physical Activity, and Metabolism. Circulation 2007; 116: 572-584.

4. Banz WJ, Maher MA, Thompson WG, Bassett DR, Moore W, Ashraf $\mathrm{M}$, et al. Effects of resistance versus aerobic training on coronary artery disease risk factors. Exp Biol Med 2003; 228: $434-440$.
RE, especially upper limb RE. However, when analyzing the HRV of volunteers who were on medication, we observed no consistent change in its behavior during the imposed loads. Even among those who were using calcium-channel blockers or ACE inhibitors as antihypertensive agents or were using amiodarone, we observed no differences throughout the loads, and the behavior of rMSSD and RMSM was the same. Although secondary analysis indicated no change in results due to the above-mentioned drugs, because of the small sample size of the study, it is not possible to state categorically that there were no drug-related changes. Thus, we considered it important to show the tendencies of these indexes, since cardiac rehabilitation programs are usually carried out with patients on several medications.

In conclusion, we can infer that, beginning at a load increment of $30 \% 1 \mathrm{RM}$, a depression in vagal modulation was produced in both groups; however, only stable CAD patients presented sympathetic overactivity at 20\% 1RM of upper limb exercise. These results provide a framework for evaluating the effects of HRV changes on upper limb exercise in this population.

\section{Acknowledgments}

The authors would like to thank all colleagues from the Cardiopulmonary Physiotherapy Laboratory and the School of Health for their friendly collaboration. More importantly, however, we are indebted to the patients for their effort and enthusiastic cooperation throughout the study. The authors would like to thank FAPESP (\#07/53202-9 and \#06/53414-3) and CAPES by way of the Interunit Post-Graduate Program in Bioengineering, University of São Paulo, for providing financial support.

5. LaFontaine $\mathrm{T}$. Resistance exercise for persons with coronary heart disease. Strength Cond J 2003; 25: 17-21.

6. Mitchell JH. J.B. Wolffe memorial lecture. Neural control of the circulation during exercise. Med Sci Sports Exerc 1990; 22: 141-154.

7. Malliani A, Montano N. Heart rate variability as a clinical tool. Ital Heart J 2002; 3: 439-445.

8. Maraes VR, Silva E, Catai AM, Novais LD, Moura MA, Oliveira $L$, et al. Identification of anaerobic threshold using heart rate response during dynamic exercise. Braz $J$ Med Biol Res 2005; 38: 731-735.

9. Lamotte M, Niset G, van de Borne P. The effect of different intensity modalities of resistance training on beat-to-beat blood pressure in cardiac patients. Eur J Cardiovasc Prev Rehabil 2005; 12: 12-17.

10. Umpierre D, Stein R. Hemodynamic and vascular effects of resistance training: implications for cardiovascular disease. Arq Bras Cardiol 2007; 89: 256-262.

11. Batagin AM, Corso SD, Soares CLR, Ferreira S, Letícia A, Souza $\mathrm{C}$, et al. Pressure response after resistance exercise 
for diferente body segments in hypertensive people. Arq Bras Cardiol 2011; 95: 405-411.

12. Simoes RP, Mendes RG, Castello V, Machado HG, Almeida LB, Baldissera V, et al. Heart-rate variability and bloodlactate threshold interaction during progressive resistance exercise in healthy older men. J Strength Cond Res 2010; 24: 1313-1320.

13. Billman GE, Kukielka M. Effects of endurance exercise training on heart rate variability and susceptibility to sudden cardiac death: protection is not due to enhanced cardiac vagal regulation. J Appl Physiol 2006; 100: 896-906.

14. Sociedade Brasileira de Cardiologia. VI Diretrizes Brasilerias de Hipertensão. Eur J Cardiovasc Prev Rehabil 2005; 14: $12-17$

15. Cooper $\mathrm{CB}$, Storer TW. Exercise testing and interpretation: A practical approach. Port Chester: Press Syndicate of the University of Cambridge; 2001.

16. Wilborn C, Greenwood M, Wyatt F, Bowden R, Grose D. The effects of exercise intensity and body position on cardiovascular variables during resistance exercise. J Exercise Physiol 2004; 7: 29-35.

17. Kraemer WJ, Fry AC. Strength testing: Development and evaluation of methodology. Physiological assessment of human fitness. Champaign: Human Kinetics; 1995.

18. Borg GA. Psychophysical bases of perceived exertion. Med Sci Sports Exerc 1982; 14: 377-381.

19. Antila K. Quantitative characterization of heart rate during exercise. Scand J Clin Lab Invest Suppl 1979; 3-68.

20. Heart rate variability: standards of measurement, physiological interpretation and clinical use. Task Force of the European Society of Cardiology and the North American Society of Pacing and Electrophysiology. Circulation 1996; 93: 1043-1065.

21. Mourot L, Bouhaddi M, Perrey S, Rouillon JD, Regnard J. Quantitative Poincare plot analysis of heart rate variability: effect of endurance training. Eur J Appl Physiol 2004; 91: 79-87.

22. Tulppo MP, Makikallio TH, Takala TE, Seppanen T, Huikuri HV. Quantitative beat-to-beat analysis of heart rate dynamics during exercise. Am J Physiol 1996; 271: H244-H252.

23. Barnard KL, Adams KJ, Swank AM, Mann E, Denny DM. Injuries and muscle soreness during the one repetition maximum assessment in a cardiac rehabilitation population. J Cardiopulm Rehabil 1999; 19: 52-58.

24. Delagardelle C, Feiereisen P, Autier P, Shita R, Krecke R, Beissel J. Strength/endurance training versus endurance training in congestive heart failure. Med Sci Sports Exerc 2002; 34: 1868-1872.

25. Gordon NF, Kohl HW III, Pollock ML, Vaandrager H, Gibbons
LW, Blair SN. Cardiovascular safety of maximal strength testing in healthy adults. Am J Cardiol 1995; 76: 851-853.

26. Braith RW, Beck DT. Resistance exercise: training adaptations and developing a safe exercise prescription. Heart Fail Rev 2008; 13: 69-79.

27. Petrofsky JS, Phillips CA, Sawka MN, Hanpeter D, Stafford D. Blood flow and metabolism during isometric contractions in cat skeletal muscle. J Appl Physiol 1981; 50: 493-502.

28. Miles DS, Cox MH, Bomze JP. Cardiovascular responses to upper body exercise in normals and cardiac patients. Med Sci Sports Exerc 1989; 21: S126-S131.

29. Sinski M, Lewandowski J, Abramczyk P, Narkiewicz K, Gaciong Z. Why study sympathetic nervous system? J Physiol Pharmacol 2006; 57 (Suppl 11): 79-92.

30. Ranpuria R, Hall M, Chan CT, Unruh M. Heart rate variability (HRV) in kidney failure: measurement and consequences of reduced HRV. Nephrol Dial Transplant 2008; 23: 444-449.

31. Hill DW, Butler SD. Haemodynamic responses to weightlifting exercise. Sports Med 1991; 12: 1-7.

32. Taaffe DR, Galvao DA, Sharman JE, Coombes JS. Reduced central blood pressure in older adults following progressive resistance training. J Hum Hypertens 2007; 21: 96-98.

33. Williamson JW, Fadel PJ, Mitchell JH. New insights into central cardiovascular control during exercise in humans: a central command update. Exp Physiol 2006; 91: 51-58.

34. Koba S, Gao Z, Xing J, Sinoway LI, Li J. Sympathetic responses to exercise in myocardial infarction rats: a role of central command. Am J Physiol Heart Circ Physiol 2006; 291: H2735-H2742.

35. Iwamoto GA, Kaufman MP. Caudal ventrolateral medullary cells responsive to muscular contraction. J Appl Physiol 1987; 62: 149-157.

36. Friedman DB, Peel C, Mitchell JH. Cardiovascular responses to voluntary and nonvoluntary static exercise in humans. $J$ Appl Physiol 1992; 73: 1982-1985.

37. Okamoto T, Masuhara M, Ikuta K. Upper but not lower limb resistance training increases arterial stiffness in humans. Eur J Appl Physiol 2009; 107: 127-134.

38. Pivarnik JM, Grafner TR, Elkins ES. Metabolic, thermoregulatory, and psychophysiological responses during arm and leg exercise. Med Sci Sports Exerc 1988; 20: 1-5.

39. Volianitis S, Secher NH. Arm blood flow and metabolism during arm and combined arm and leg exercise in humans. J Physiol 2002; 544: 977-984.

40. Castanheira-Neto AG, Costa-Filho IR, Farinatti PTV. Cardiovascular responses to resistance exercise are affected by workload and intervals between sets. Arq Bras Cardiol 2010; 95: 493-501. 